\title{
Tumor suppressive activity of prolyl isomerase Pin1 in renal cell carcinoma
}

\author{
Brian L. Teng ${ }^{a}$, Kathryn E. Hacker ${ }^{b}$, Shufen Chen $^{b}$, Anthony R. Means ${ }^{a}$, W. Kimryn \\ Rathmell ${ }^{b, *}$ \\ ${ }^{a}$ Department of Pharmacology and Cancer Biology, Duke University, 308 Research Drive, Durham, NC 27710, USA \\ bineberger Comprehensive Cancer Center, University of North Carolina at Chapel Hill, 450 West Drive CB 7295, Chapel Hill, \\ NC 27599-7295, USA
}

\section{A R T I C L E I N F O}

Article history:

Received 20 May 2011

Received in revised form

21 June 2011

Accepted 22 June 2011

Available online 28 June 2011

Keywords:

Pin1

Prolyl isomerase

Renal cell carcinoma

Conditional tumor suppressor

\begin{abstract}
A B S T R A C T
Pin1 specifically recognizes and catalyzes the cis-trans isomerization of phosphorylated-Ser/ Thr-Pro bonds, which modulate the stability, localization, and function of numerous Pin1 targets involved in tumor progression. However, the role of Pin 1 in cancer remains enigmatic as the gene is located on chromosome 19p13.2, which is a region subject to loss of heterozygosity in several tumors. Since Pin1 protein is frequently under-expressed in kidney cancer, we have explored its role in human clear cell renal cell carcinoma (ccRCC). Here we show evidence for PIN1 gene deletion and mRNA under-expression as a mechanism of Pin1 reduction in CCRCC tumors. We demonstrate that restoration of Pin1 in cell lines found to be deficient in Pin1 protein expression can attenuate the growth of ccRCC cells in soft agar and a xenograft tumor model. Moreover, this ability of Pin1 to negatively influence tumor growth in ccRCC cells may be dependent on the presence of functional p53, which is infrequently mutated in ccRCC. These observations suggest Pin1 may have a mild tumor suppressive role in ccRCC.
\end{abstract}

(c) 2011 Federation of European Biochemical Societies. Published by Elsevier B.V. All rights reserved.

\section{Introduction}

Pin1 is a conserved peptidyl-prolyl isomerase that specifically recognizes phosphorylated serine/threonine-proline motifs (pS/T-P) (Yaffe et al., 1997). By catalyzing the cis-trans isomerization of $\mathrm{pS} / \mathrm{T}$-P bonds, Pin1 induces conformational changes in its target phosphoproteins that can alter their biological function and/or stability (Lu and Zhou, 2007). Several proteins important for cell cycle and cancer progression are regulated by Pin1, including $\beta$-catenin, c-Jun, c-Myc, cyclin D1, cyclin E, and p53 (Liou et al., 2002; Ryo et al., 2001; Wulf et al., 2001; Yeh et al., 2004, 2006; Zacchi et al., 2002; Zhenget al., 2002). Since these targets are key regulators of G1-S progression, Pin1 has been suggested to function as a molecular timer for the cell cycle (Yeh and Means, 2007). Indeed, the loss of Pin1 in multiple cell types, such as Pin1 ${ }^{-/-}$primordial germ cells and mouse embryonic fibroblasts (MEF), leads to prolongation of G0-G1-S progression (Atchison et al., 2003; Fujimori et al., 1999; You et al., 2002). Such a delay could be explained by Pin1's ability to promote the expression and stabilization of cyclin D1, underscoring the potential importance of Pin1 in cancer.

A number of studies report prevalent Pin1 over-expression in human cancers, including those of breast and prostate (Ayala et al., 2003; Bao et al., 2004; Wulf et al., 2001). In breast

Abbreviations: ccRCC, clear cell renal cell carcinoma; MEF, mouse embryonic fibroblasts; RPTEC, renal proximal tubule epithelial cells; GSA, gene set analysis; NSC, non-silencing control shRNA; shPin1, Pin1 shRNA.

* Corresponding author. Tel.: +1 919966 8644; fax: +1 9199668212.

E-mail address: rathmell@med.unc.edu (W.K. Rathmell).

1574-7891/\$ - see front matter @ 2011 Federation of European Biochemical Societies. Published by Elsevier B.V. All rights reserved. doi:10.1016/j.molonc.2011.06.002 
cancer, Pin1 over-expression correlates with the deregulation of cyclin D1, which is likely the result of enhanced transcription of this gene by c-Jun and $\beta$-catenin as well as posttranslational stabilization of the protein (Ryo et al., 2001; Wulf et al., 2001). Pin1 levels also correlate with poor clinical outcome and may have prognostic value in the case of prostate cancer (Ayala et al., 2003). Based on these studies, one might conclude that increased levels of Pin1 promote tumor progression. However, and perhaps not surprising given the plethora of Pin 1 targets, the role of Pin 1 in human cancer is complex and context dependent.

In fact, several reports suggest Pin1 might inhibit tumor progression by destabilizing oncoproteins (i.e. cyclin E, c-Myc) or by activating tumor suppressors (i.e. p53, p73) (Yeh and Means, 2007). Previously, we showed that cyclin E is stabilized in Pin $1^{-1-}$ C57BL6 MEF, which correlated with defective cell cycle progression and accelerated genomic instability (Yeh et al., 2006). Moreover, Pin $1^{-/-}$MEF cells transformed with $\mathrm{p} 53^{\mathrm{DD}}$ and/or H-Ras ${ }^{\mathrm{G} 12 \mathrm{~V}}$ when grown as xenografts displayed reduced tumor free survival and increased tumor growth compared to wild type MEF, which suggests Pin1 may exhibit a tumor suppressive effect in these cells. Since Pin1 appears to play a tumor promoting or inhibitory role depending on the genetic context, Pin1 has been described as a "conditional" tumor suppressor (Yeh and Means, 2007). However, Pin1-mediated tumor suppressive effects in a human context have yet to be demonstrated.

Interestingly, Pin 1 is prevalently under-expressed in some human cancers, including kidney cancer (Bao et al., 2004). Although the relevance of Pin1 under-expression in kidney cancer has not been evaluated, the effect of differential Pin1 levels on tumor progression likely depends on the specific repertoire of genetic mutations acquired by the cancer cells. In particular, although p53 is commonly mutated in many human cancers, p53 mutation is infrequent in renal cell carcinoma (RCC) (Dalgliesh et al., 2010; Gurova et al., 2004). Since Pin1 has been shown to positively regulate $\mathrm{p} 53$, one hypothesis is that Pin1 can exert a tumor suppressive role in a manner dependent on wild type p53. Herein, we evaluate the role of Pin1 in RCC and find support for this hypothesis.

\section{Materials and methods}

\subsection{Copy number analysis}

SNP6.0 array data corresponding to gene expression data deposited in Gene Expression Omnibus GSE17818 (Dalgliesh et al., 2010) were obtained from the Wellcome Trust Sanger Institute (Hinxton, Cambridge, UK). Raw allele intensities were imported into Partek Genomics Suite software, version 6.5 (St. Louis, MO, USA). Based on the PCA plot, one outlying tumor and corresponding normal were excluded, batch effect was removed, and copy number values were created from allele intensities. Genomic segmentation was performed with segmentation parameters of a minimum of 10 genomic markers, $p$-value threshold of 0.001 , and a signal to noise ratio of 0.3. Identified regions were visualized using the Plot Chromosome function within Partek Genomic Suite.

\subsection{Gene expression analysis}

PIN1 gene expression levels were examined from previously described gene expression data (Brannon et al., 2010), which was re-downloaded with 44 additional new tumor samples from the UNC Microarray Database in log2 Lowess-normalized sample/reference format (median), filtered for $70 \%$ of probes present above background levels and annotated using the 20101031 Agilent annotation release. The data was imported into Partek Genomics Suite software and batch effect was removed through Partek's batch effect removal algorithms. All human studies were approved by the University of North Carolina Biomedical Institutional Review Board and Office of Human Research Ethics.

\subsection{Cell lines and antibodies}

Primary human renal proximal tubule epithelial cells (RPTEC) were cultured in REGM Renal Epithelial Cell Growth Medium (Lonza, Walkersville, MD, USA). Human renal cell carcinoma ACHN and A498 cells, and human embryonic kidney 293T cells (American Type Culture Collection, Manassas, VA, USA) were cultured in Dulbecco's modified Eagle's medium (Mediatech, Manassas, VA, USA) supplemented with $10 \%$ fetal bovine serum (Gemini Bio-Products, West Sacramento, CA, USA) and 1\% penicillin-streptomycin (Mediatech). Cells were maintained at $37^{\circ} \mathrm{C}$ in a $5 \% \mathrm{CO}_{2}$ humidified incubator. The following antibodies were used: anti-Pin1 (Winkler et al., 2000), anti- $\beta$-actin (Sigma-Aldrich, St. Louis, MO, USA), anti-Ki67 (Lab Vision, Fremont, CA, USA), IRDye800-conjugated anti-mouse IgG (Rockland Immunochemicals, Gilbertsville, PA, USA), and Alexa Fluor 680-conjugated anti-rabbit IgG (Invitrogen, Carlsbad, CA, USA).

\subsection{Retrovirus preparation and transduction}

Pin1 shRNA construct was generated by inserting a sequence targeting 5'-CTGCCACCGTCACACAGTA-3' into pSUPER. Nonsilencing control shRNA construct pSUPER. Mamm-X was obtained from Oligoengine (Seattle, WA, USA). Pin1 expression construct was created by PCR amplifying the human Pin1 cDNA from pGEX-2TK-Pin1 (Yi et al., 2005) and ligating it into the EcoRI-SalI sites of pBABE-neo. Site-directed mutagenesis of $\mathrm{pBABE}-\mathrm{neo}-\mathrm{Pin} 1^{\mathrm{WT}}$ to $\mathrm{Pin} 1^{\mathrm{W} 34 \mathrm{~A}}$ was carried out by GenScript (Piscataway, NJ, USA). pBABE-puro-p53 ${ }^{\mathrm{DD}}$ construct was created by excising p53 ${ }^{\mathrm{DD}}$ from pBABE-hygro-p53 ${ }^{\mathrm{DD}}$ (Yeh et al., 2006), then inserting it into the BamHI-EcoRI sites of pBABE-puro. Retroviruses were produced in 293T cells following established methods (O'Hayer and Counter, 2006). ACHN cells were infected with retrovirus, then stably selected using $1 \mathrm{mg} / \mathrm{ml} \mathrm{G} 418$ (Invitrogen) or $2 \mu \mathrm{g} / \mathrm{ml}$ puromycin (Sigma-Aldrich) for 7-10 days.

\subsection{Immunoblotting}

Equal amounts of protein sample were resolved using SDS-PAGE and then transferred to Immobilon-FL membrane (Millipore, Billerica, MA, USA). Quantitative immunoblotting was carried out using procedures established by LI-COR Biosciences (Lincoln, NE, USA). 


\subsection{RT-PCR}

RNA was isolated using RNeasy Plus Mini Kit (Qiagen, Valencia, CA, USA). Transgenic expression of $\mathrm{p} 53^{\mathrm{DD}}$ was verified by RT-PCR as previously described (Adam and Counter, 2008).

\subsection{Soft agar assay}

Cellular transformation was evaluated by soft agar assay as previously described (O'Hayer and Counter, 2006). Briefly, $2.5 \times 10^{4}$ ACHN cells were seeded per well of a 6 -well plate. Cells were fed with $0.2 \mathrm{ml}$ of culture media every 3-4 days for 21 days. Colonies greater than 30 cells were counted under a light microscope. Images were acquired with a Sony DSCW50 (New York, NY, USA) attached to a Zeiss Axiovert 10 Microscope (Oberkochen, Germany) using a CamAdapter Kit (The Microscope Store, Wirtz, VA, USA).

\subsection{Xenograft tumor model}

Five hundred thousand or $10^{6}$ ACHN cells were subcutaneously injected into each flank of athymic nude mice using protocols approved by the University of North Carolina Institutional Animal Care and Use Committee. Tumor dimensions were measured every 3-4 days with calipers until largest tumors reached $0.8 \mathrm{~cm}$ in length, after which the animals were sacrificed. Xenograft tumors were fixed with formalin and embedded in paraffin for tissue sectioning.

\subsection{Immunohistochemistry}

Immunohistochemical staining was performed as previously published (Chen et al., 2010). TUNEL assay was performed using ApopTag Peroxidase In Situ Apoptosis Detection Kit (Millipore). High resolution images were captured using an Infinity 2-3 Camera (Lumenera Corporation, Ottawa, ON, Canada) affixed to an Olympus CX41 Microscope (Center Valley, PA, USA). No primary antibody control images are provided in Supplementary Figure 3. Average percent DAB-positive staining was quantified from 10 different fields per tumor using ImmunoRatio (Tuominen et al., 2010) or blinded manual cell counting.

\subsection{Statistical analysis}

Bar graphs show mean \pm standard error of the indicated number of experiments or samples. Statistical analyses were performed using Microsoft Excel and StatView. Cutoff for statistical significance was arbitrarily set at $P<0.05$. $^{*}$ denotes significant and ${ }^{* *}$ denotes highly significant, with specific $P$ values indicated in the figure legends.

\section{Results}

\subsection{PIN1 gene is frequently deleted and under-expressed in human clear cell RCC (ccRCC)}

Previous studies indicate that the Pin 1 protein is prevalently under-expressed in kidney tumors compared to normal kidney tissue (Bao et al., 2004). As shown in Figure 1A, we determined that Pin1 under-expression in kidney cancer may be in part due to deletion of the PIN1 locus from chromosome 19p13.2, and resultant haploinsufficiency, as copy number analysis revealed Pin1 loss in 24 of 86 (28\%) ccRCC tumors. In order to evaluate the effective mRNA expression level of the PIN1 gene, we examined 18 renal tumor and normal kidney paired samples for relative PIN1 gene expression. We observed that the PIN1 gene was under-expressed in 4 of $18(22 \%)$ ccRCC tumors relative to paired normal kidney tissue (Figure 1B), which correlates with the frequency of PIN1 deletion observed in ccRCC. Since genetic loss of function is a hallmark of tumor suppressors, we further assessed the relevance of Pin1 under-expression in ccRCC by gene set analysis (GSA). GSA revealed low Pin1 levels correlate with reduced expression of genes involved in p53 stabilization as well as mitotic cell cycle pathways (Supplementary Figure 1). In addition, depressed Pin1 levels are associated with increased expression of genes involved in metastasis, stem cell-related, and proliferation pathways (Supplementary Figure 1). Collectively, these observations are compatible with the hypothesis that Pin1 may have tumor suppressive function in ccRCC.

\subsection{Pin1 attenuates growth of ccRCC cells in soft agar}

To test if Pin1 could serve a tumor suppressive role, we selected two RCC cell lines that displayed reduced expression of Pin1 protein, ACHN and A498 (Figure 2A; Supplementary Figure $2 \mathrm{~A}$ ). The rescue of reduced Pin1 levels to $\sim 1.5$-fold normal levels with ectopic expression of Pin1 was sufficient to modestly, but significantly inhibit the growth of both ACHN and A498 cells in soft agar (Fig. 2A-C; Supplementary Figure 2). Since A498 cells formed fewer and much smaller colonies, ACHN cells were chosen for further analysis of Pin1mediated effects. To evaluate the importance of Pin1's WWdomain in tumor suppressive activity, ACHN cells were transduced with Pin $1^{\mathrm{W} 34 \mathrm{~A}}$, a WW-domain mutant incapable of binding to and isomerizing Pin1 substrates (Lu et al., 1999). Although Pin $1^{\mathrm{W} 34 \mathrm{~A}}$ was expressed at a level similar to Pin $1^{\mathrm{WT}}$ (Figure $2 \mathrm{~A}$ ), Pin $1^{\text {W34A }}$ was unable to diminish the growth of ACHN cells in soft agar (Figure 2B, C). This result suggests a functional WWdomain and canonical activity of Pin1 are essential for Pin1mediated inhibition of anchorage independent growth. Conversely to ectopic Pin1 expression, further Pin1 depletion from ACHN cells by shRNA (Figure 2D) was associated with a $47 \%$ increase in soft agar colony formation (Figure 2E, F). These data demonstrate Pin1 can impede the growth of ccRCC cells in soft agar, which is consistent with tumor suppressive activity.

\subsection{Pin1 attenuates ACHN tumor growth in a xenograft model}

To further examine the role of Pin1 in tumor growth, ACHNNeo (vector control) and ACHN-Pin1 cells rescued with ectopic Pin1 expression were assayed for tumorigenicity in a xenograft model. Consistent with our soft agar assay data, Pin1 expression significantly improved tumor free survival and suppressed the tumorigenic growth of ACHN xenografts (Figure 3A, B). ACHN-Pin1 tumors were histologically similar to ACHN-Neo control tumors, which exhibited necrotic cores surrounded by proliferating cells (Figure 3C). In addition, 
A

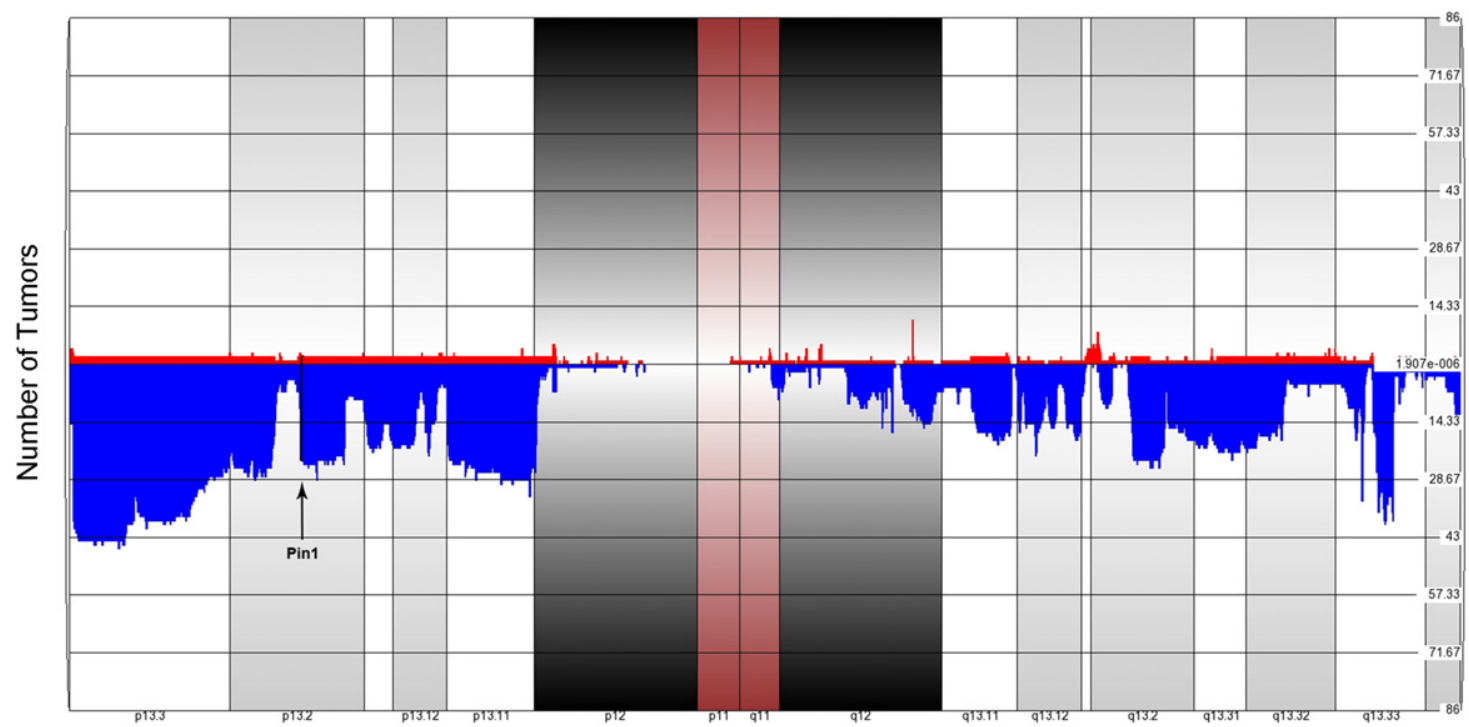

Location on Chromosome 19

B

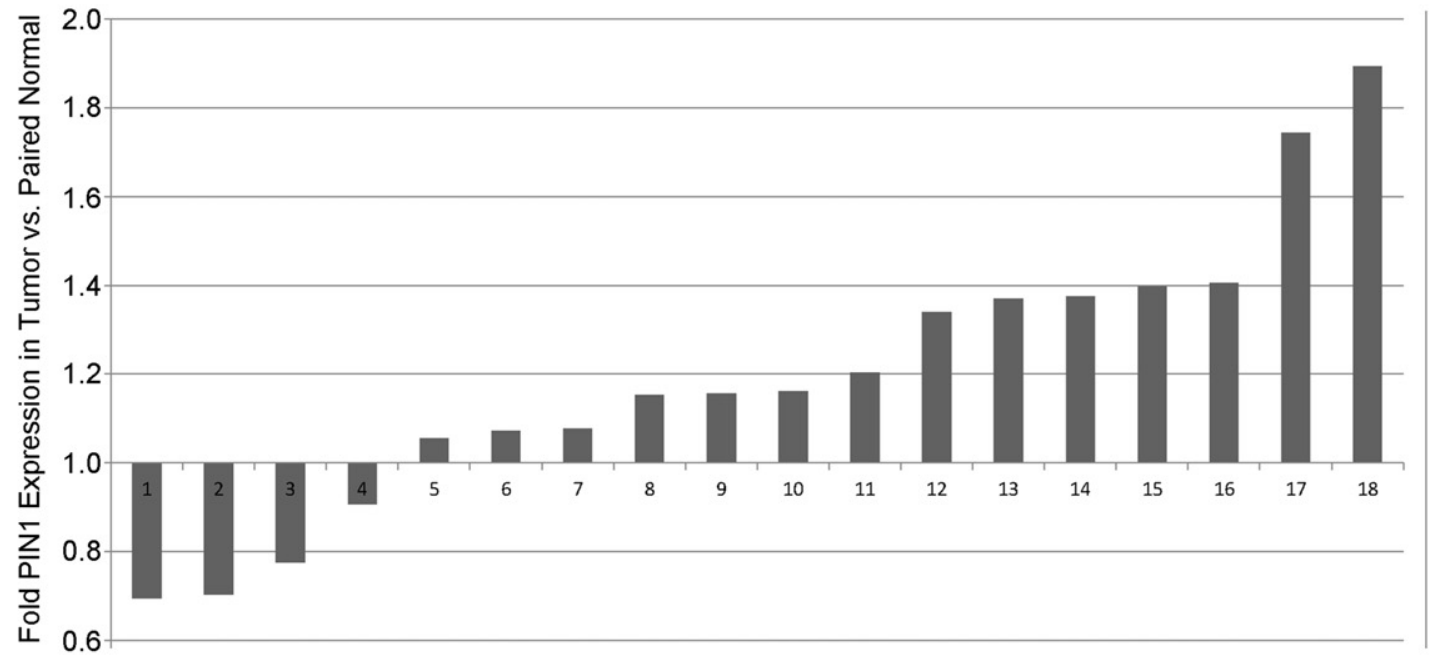

ccRCC Tumor Sample

Figure 1 - PIN1 gene is frequently deleted and under-expressed in human ccRCC tumors. (A) Frequency of tumors containing deletion (blue) or amplification (red) of each region of chromosome 19. The PIN1 gene on 19p13.2 (9945999...9960358) is highlighted in black. (B) Fold PIN1 gene expression in individual ccRCC tumor samples relative to matched normal kidney tissue, as determined by gene expression microarray.

Pin1 immunohistochemical analysis showed ACHN-Pin1 tumors retained elevated levels of Pin1 (Figure 3D), and thus did not grow as a result of escaping Pin1 over-expression.

To explore the means by which Pin1 expression impeded xenograft growth, we compared rates of proliferation and apoptosis in the xenografts. Ectopic Pin1 expressing tumors displayed significantly reduced Ki67 staining, indicating that Pin1 contributes to decreased RCC tumor growth by inhibiting cellular proliferation (Figure 4A). Indeed, ACHN-Pin1 tumors showed a $29 \%$ decrease in the number of Ki67-positive nuclei (Figure 4C), which is comparable to the impaired growth of ACHN-Pin1 cells in soft agar (Figure $2 \mathrm{C}$ ), and is consistent with our GSA showing that Pin1 negatively correlates with genes involved in proliferation (Supplementary Figure 1). Intriguingly, ectopic rescue of Pin1 also led to increased apoptosis in the xenograft tumors as measured by TUNEL staining (Figure 4B, D). Thus ectopic Pin1 expression attenuates ACHN tumor growth through effects on both proliferation and apoptosis.

\subsection{Repression of p53 activity can prevent Pin1- mediated tumor inhibitory activity}

Since previous research suggests Pin1 can enhance p53 function in order to inhibit cell proliferation and induce 
A

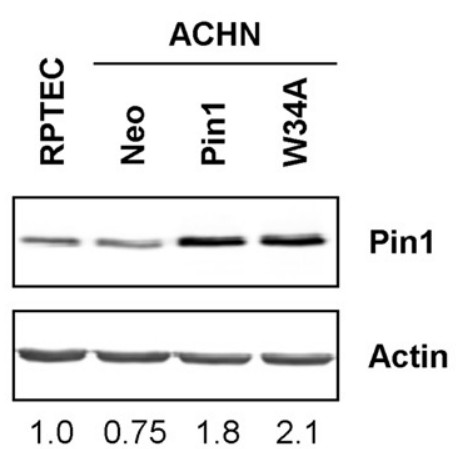

B
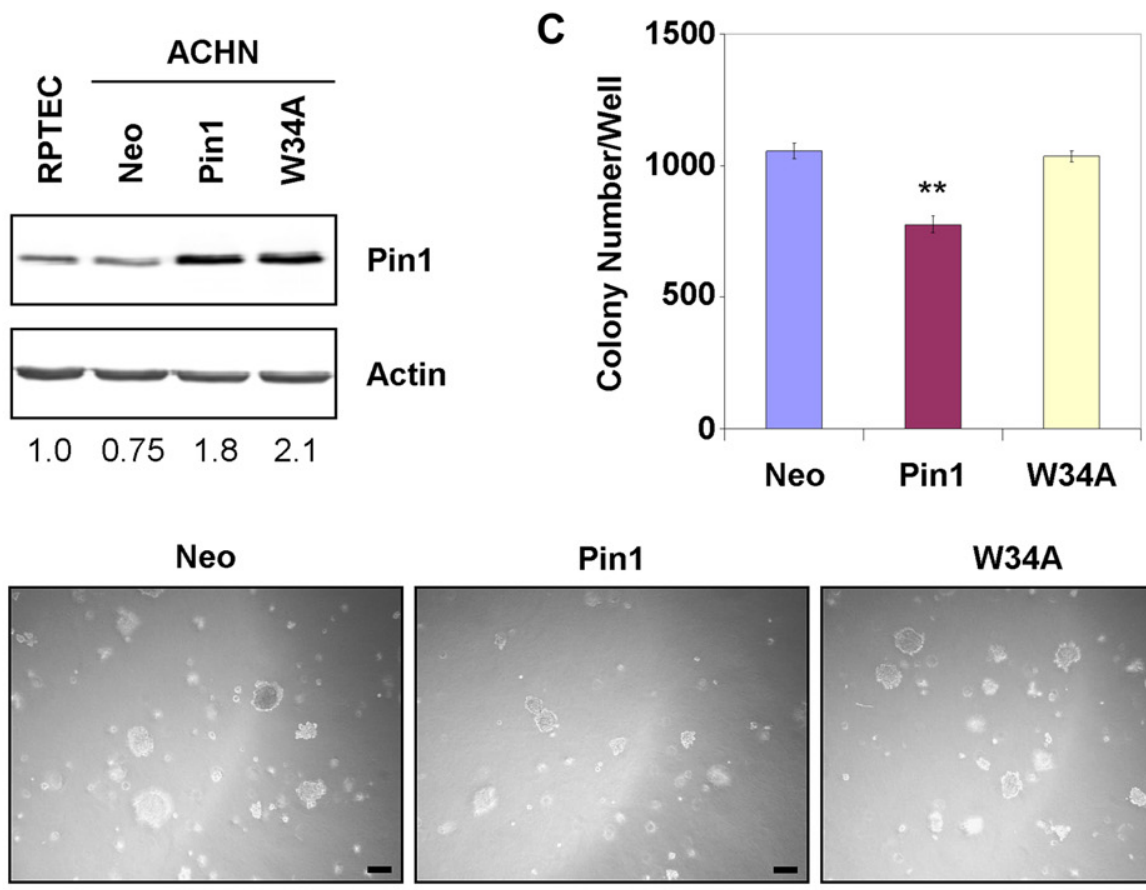

D

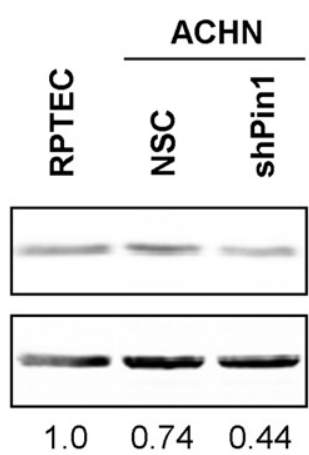

Pin1

Actin

E

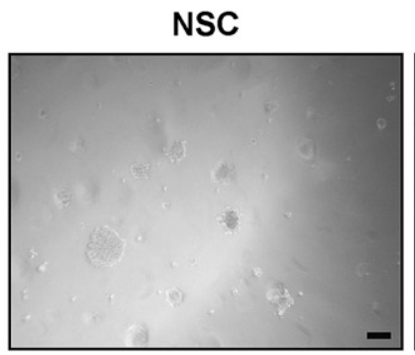

F

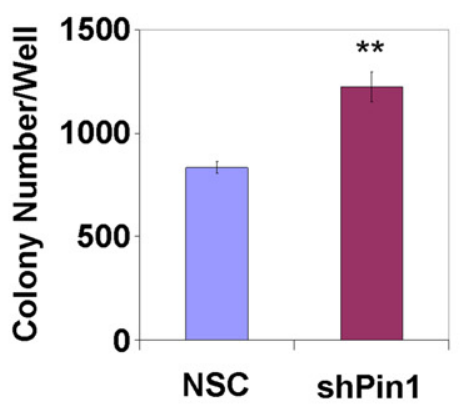

shPin1

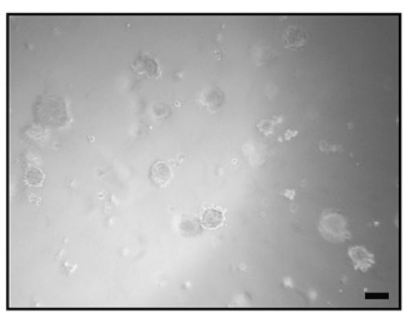

Figure 2 - Pin1 attenuates growth of human renal cell carcinoma ACHN cells in soft agar. (A) RPTEC or ACHN cells transduced with empty vector (Neo), Pin1 or Pin1 ${ }^{\text {W34A }}$ were analyzed by immunoblot. Average normalized Pin1 levels relative to RPTEC are below. (B, C) ACHN-Neo, ACHN-Pin1, and ACHN-W34A cells were grown in soft agar. After 21 days, colonies were quantified from three independent assays done in triplicate. (D) RPTEC or ACHN cells transduced with non-silencing control shRNA (NSC) or Pin1 shRNA (shPin1) were analyzed by immunoblot as in (A). (E, F) ACHN-NSC and ACHN-shPin1 cells were assayed for growth in soft agar as in (B, C), except results represent two independent experiments performed in triplicate. Scale bars represent $125 \mu \mathrm{m}{ }^{* *} P<0.001$ vs. control, $t$-test.

apoptosis in response to genotoxic stress (Zacchi et al., 2002; Zheng et al., 2002), we hypothesized that the Pin1-mediated growth inhibitory effect on tumors might require functional p53, which is known to be wild type in ACHN cells (O'Connor et al., 1997; Warburton et al., 2005). To test this hypothesis, p53 was specifically inhibited in ACHN-Pin1 cells by a dominant negative p53 (p53 ${ }^{\mathrm{DD}}$ ) mutant that we have previously utilized (Yeh et al., 2006). Expression of $\mathrm{p} 53^{\mathrm{DD}}$ and Pin1 was verified by RT-PCR or immunoblot, respectively (Figure 5A, B). As shown in Fig. 5C-E, as expected, 
A

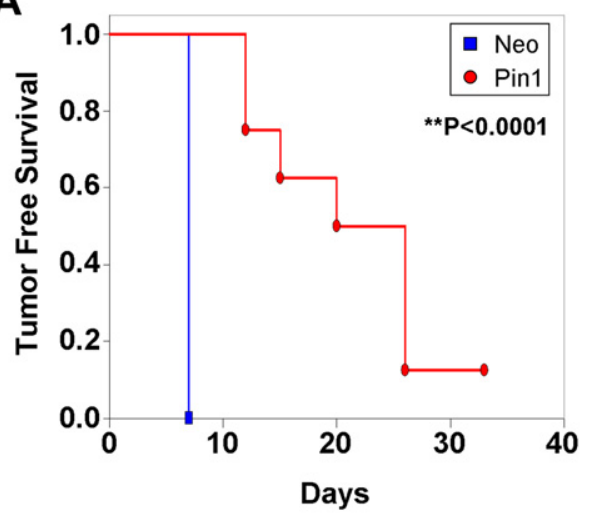

C

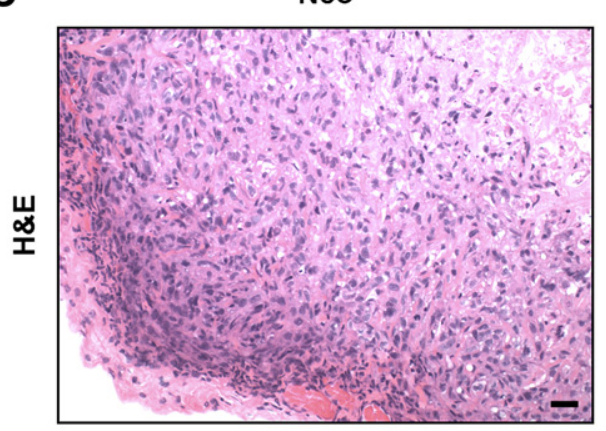

D

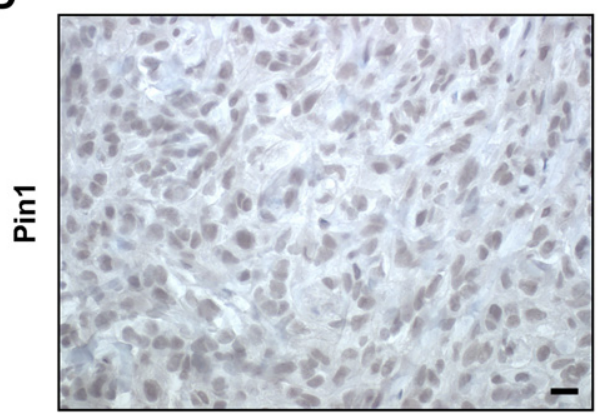

B

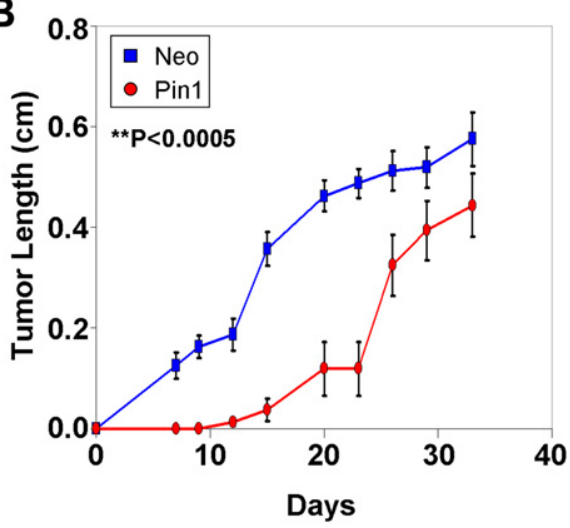

Pin1
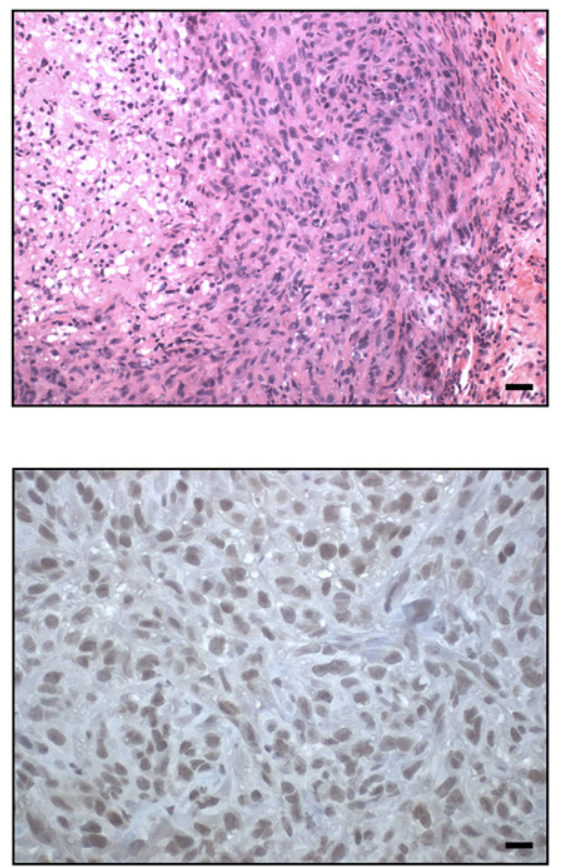

Figure 3 - Pin1 attenuates tumor growth of ACHN cells in xenograft model. ACHN-Neo and ACHN-Pin1 cells were subcutaneously injected into each flank of athymic nude mice. (A) Tumor free survival of ACHN-Neo $(n=8)$ and ACHN-Pin1 $(n=8)$ xenografts was assessed by Kaplan-Meier survival analysis. $P$-value determined by log-rank test. (B) Tumor growth was assessed from measurements of tumor length. $P$-value determined by repeated measures ANOVA. (C) Hematoxylin and eosin (H\&E) staining. Scale bars represent $50 \mu \mathrm{m}$ (D) Pin1 was detected by DAB immunohistochemistry (IHC) then counterstained with hematoxylin. Scale bars represent $25 \mu \mathrm{m}$.

the inhibition of $\mathrm{p} 53$ by $\mathrm{p} 53^{\mathrm{DD}}$ enhanced growth in soft agar and in xenograft; however, ectopic Pin1 rescue in this setting failed to suppress soft agar colony formation or xenograft tumor growth. Therefore Pin1-mediated tumor inhibitory activity in RCC appears to require functional p53.

\section{Discussion}

Here we provide the first demonstration of Pin1-mediated tumor inhibitory activity in human cancer cells. The restoration of Pin1 expression in Pin1-deficient ccRCC cells produced reductions in tumor cell growth in soft agar, as well as in xenograft model growth. These effects although modest, were significant, and would be expected to have substantial consequences over the long natural history of renal cell carcinoma. Because tumorigenicity was not ablated, we have referred to Pin 1 as a protein with tumor inhibitory activity, rather than a nascent tumor suppressor. Moreover, Pin1's tumor inhibitory effects in renal carcinoma cells seem to require functional p53, as p53 inhibition was sufficient to prevent Pin1-mediated tumor suppressive activity in these cells. This result suggests the possibility that Pin 1 acts to attenuate tumor cell growth in conditions in which p53 signaling is intact, and is consistent with the physiologic role of Pin 1 in positively regulating p53 biological function, which has been previously 
A

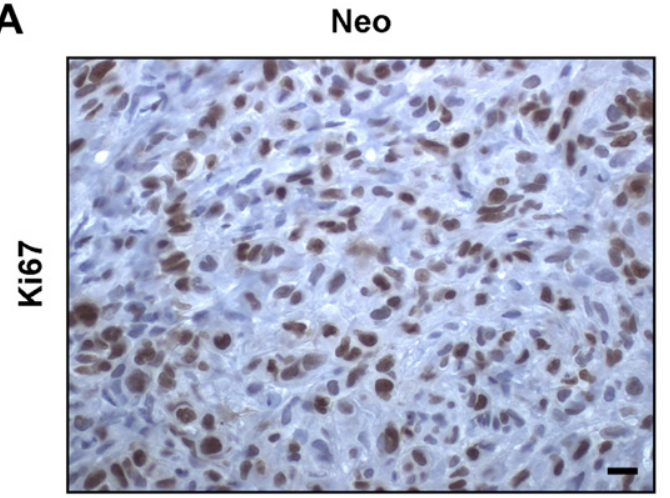

B

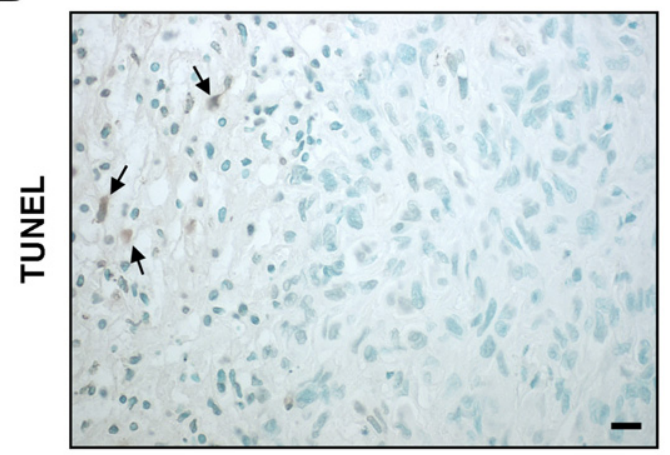

Pin1
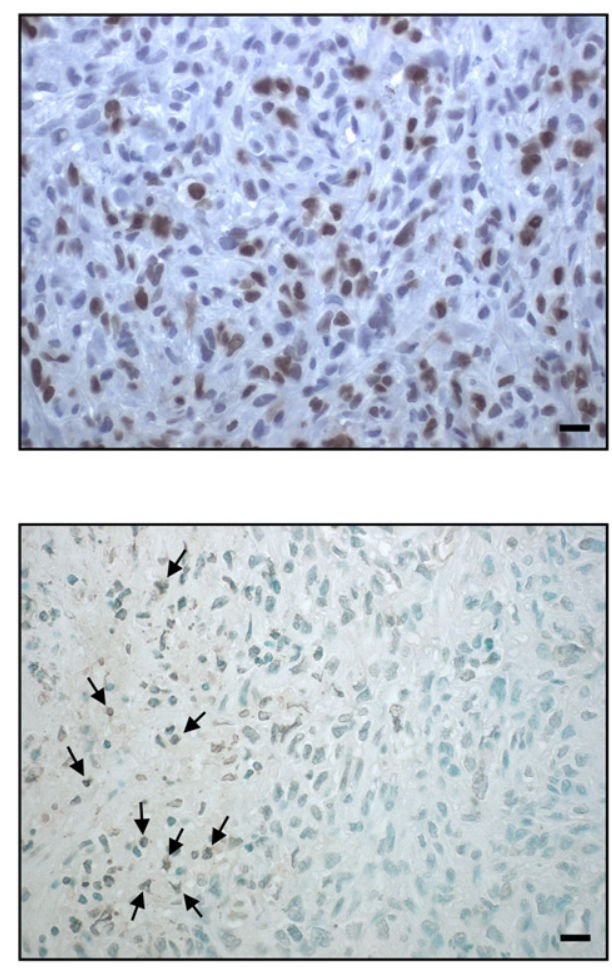

C

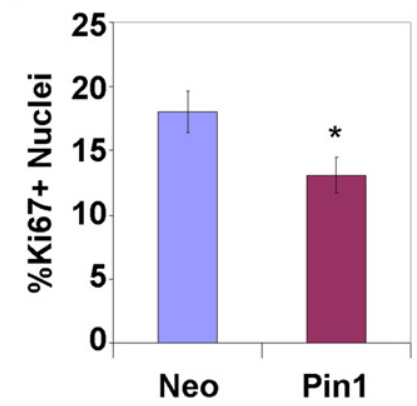

D

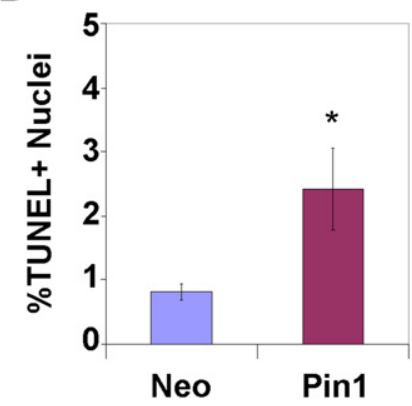

Figure 4 - Pin1 attenuates ACHN xenograft tumor growth through effects on proliferation and apoptosis. (A) Proliferation was assessed by IHC analysis of Ki67. (B) Apoptosis was evaluated by TUNEL assay. Black arrows point to examples of DAB-positive nuclei. (C, D) Average percent DAB-positive nuclei was quantified for (A) Ki67 and (B) TUNEL stains respectively. Data represent ACHN-Neo $(n=8)$ and ACHN-Pin1 $(n=7)$ with ${ }^{*} P<0.05$ vs. control, $t$-test. Scale bars represent $25 \mu \mathrm{m}$.

documented (Berger et al., 2005; Mantovani et al., 2007; Zacchi et al., 2002; Zheng et al., 2002). As the PIN1 gene is frequently deleted in ccRCC tumors and Pin1 exhibits tumor attenuating activity in p53 wild type ccRCC cell lines, but not those in which p53 is inhibited, we surmise that Pin1 may play an important role in cancer biology to conditionally inhibit growth in some scenarios while exerting tumor promoting activities in other contexts.

Certainly, the concept of a protein functioning with conditional tumor suppressive activity is not novel. Indeed, there are several proteins reported to possess tumor suppressing or promoting activity depending on the genetic context, including deleted in colon cancer (DCC), netrin-1 and transforming growth factor $\beta$ (TGF $\beta$ ) (Massagué, 2008; Mazelin et al.,
2004). In "normal" contexts, TGF $\beta$ mediates tumor suppression through mechanisms that regulate cytostasis, apoptosis, and differentiation. However, TGF $\beta$ can alternatively promote the growth and metastasis of tumors containing certain genetic mutations or deletions that disable TGF $\beta$ 's tumor suppressive function. The example of TGF $\beta$ interestingly seems to share some parallels with what has been observed for Pin1 in cancer. Since Pin1 has been shown to function to either decrease or enhance tumor growth, we speculate that mutations that disable Pin1's tumor suppressing activities (i.e. p53 mutation) may enhance Pin1's tumor promoting activities (i.e. cyclin D1 over-expression) in these contexts. Notably, frequent Pin1 over-expression correlating with high cyclin D1 expression has been shown to occur in human cancers where 
A

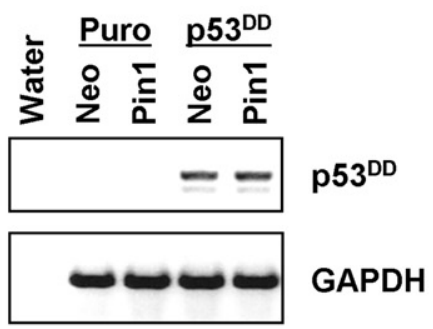

C
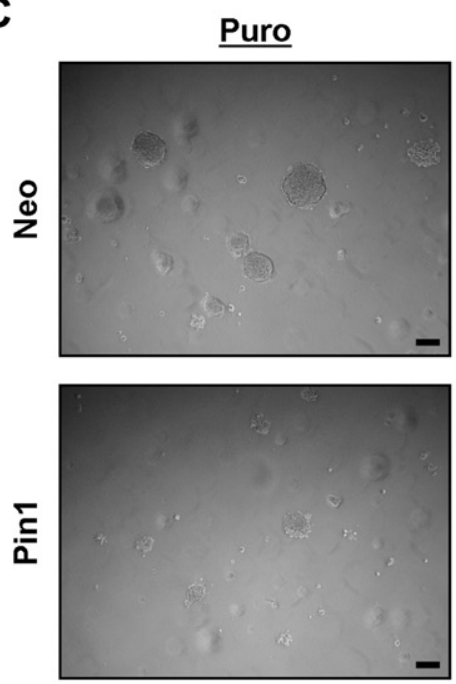

D

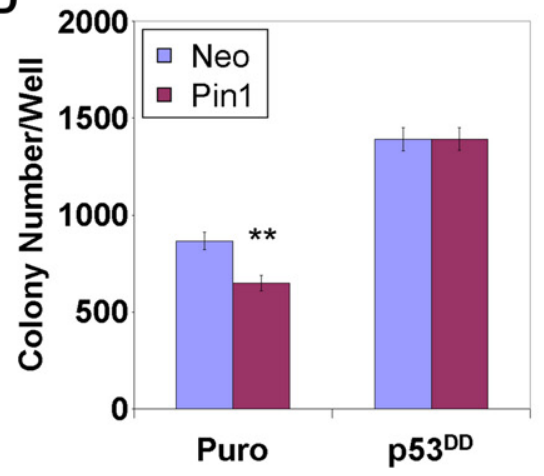

B

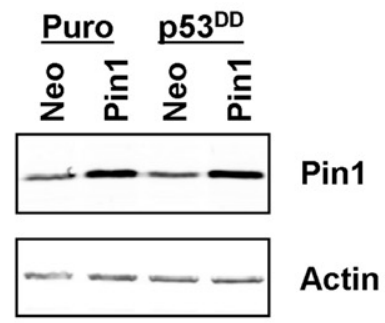

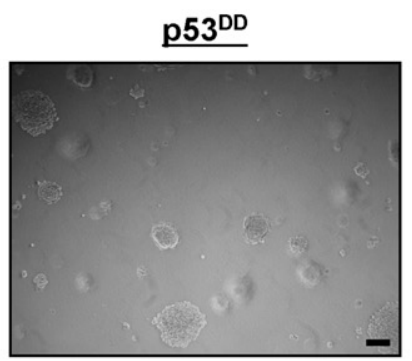

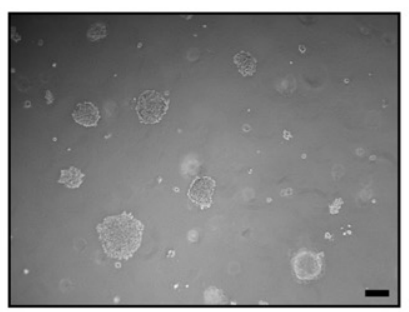

E

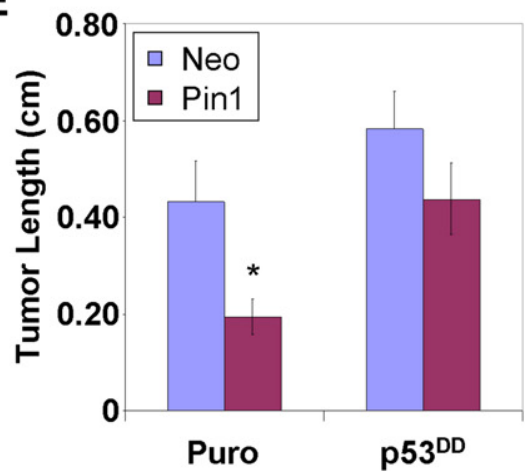

Figure 5 - Repression of p53 blocks Pin1-mediated tumor suppressive activity in ACHN cells. (A) ACHN cells transduced with the indicated combinations of vector (Neo, Puro), Pin1, and $\mathrm{p}^{\mathrm{DD}}$ were analyzed for $\mathrm{p}^{\mathrm{DD}}{ }^{\mathrm{DD}}$ expression by RT-PCR. (B) Ectopic Pin1 expression in cell lines from (A) was confirmed by immunoblot. (C, D) ACHN cells from (A, B) were assayed for growth in soft agar with results quantified from two independent experiments performed in triplicate. Scale bars represent $125 \mu \mathrm{m}$. (E) Final tumor length of ACHN xenograft tumors $(n=8)$ grown for four months. ${ }^{*} P<0.05$ or ${ }^{* *} P<0.005$ vs. corresponding Neo control, $t$-test.

p53 is frequently mutated, including breast and liver cancers (Pang et al., 2004; Wulf et al., 2001).

On the other hand, frequent Pin1 under-expression has been most commonly observed in cancers where p53 is frequently wild type, including kidney and testicular cancers (Bao et al., 2004; Gurova et al., 2004; Heimdal et al., 1993). Based on these observations, the role of Pin1 as an inhibitor of human tumorigenesis seems to be highly dependent on the tumor cell context. In CCRCC which harbors infrequent mutations of p53 and other common mediators of tumorigenesis, Pin1 appears to play a mild tumor suppressive role, and further investigation is warranted to ascertain if Pin 1 could be a useful prognostic factor or therapeutic target in human cancer.

\section{Conflict of interest}

The authors declare no conflict of interest. 


\section{Acknowledgments}

We thank Christopher Counter for providing pBABE and pSUPER plasmids. Thank you to Rose Brannon for hours of helpful advice. We also thank Charlene M. Ross and the UNC Animal Studies Core Facility for assisting in the xenograft studies. This research was supported by R01 CA082845 (ARM) and R01 CA121781 (WKR). Brian Teng received training support through a Department of Defense Breast Cancer Research Program Pre-Doctoral Fellowship (W81XWH-06-10442). Kathryn Hacker received support from the UNC Department of Genetics T32 Training grant.

\section{Appendix Supplementary data.}

Supplementary data associated with this article can be found, in the online version, at doi:10.1016/j.molonc.2011.06.002.

\section{R E F E R E N C E S}

Adam, S.J., Counter, C.M., 2008. A method to generate genetically defined tumors in pigs. Methods Enzymol., 39-51. Elsevier.

Atchison, F.W., Capel, B., Means, A.R., 2003. Pin1 regulates the timing of mammalian primordial germ cell proliferation. Development 130, 3579-3586.

Ayala, G., Wang, D., Wulf, G., Frolov, A., Li, R., Sowadski, J., Wheeler, T.M., Lu, K.P., Bao, L., 2003. The prolyl isomerase Pin1 is a novel prognostic marker in human prostate cancer. Cancer Res. 63, 6244-6251.

Bao, L., Kimzey, A., Sauter, G., Sowadski, J.M., Lu, K.P., Wang, D.G., 2004. Prevalent overexpression of prolyl isomerase Pin1 in human cancers. Am. J. Pathol. 164, 1727-1737.

Berger, M., Stahl, N., Del Sal, G., Haupt, Y., 2005. Mutations in proline 82 of p53 impair its activation by Pin1 and Chk2 in response to DNA damage. Mol. Cell Biol. 25, 5380-5388.

Brannon, A.R., Reddy, A., Seiler, M., Arreola, A., Moore, D.T., Pruthi, R.S., Wallen, E.M., Nielsen, M.E., Liu, H., Nathanson, K.L., 2010. Molecular stratification of clear cell renal cell carcinoma by consensus clustering reveals distinct subtypes and survival patterns. Genes Cancer 1, 152-163.

Chen, S., Sanford, C.A., Sun, J., Choi, V., Van Dyke, T., Samulski, R.J., Rathmell, W.K., 2010. VHL and PTEN loss coordinate to promote mouse liver vascular lesions. Angiogenesis 13, 59-69.

Dalgliesh, G.L., Furge, K., Greenman, C., Chen, L., Bignell, G., Butler, A., Davies, H., Edkins, S., Hardy, C., Latimer, C., 2010. Systematic sequencing of renal carcinoma reveals inactivation of histone modifying genes. Nature 463, 360-363.

Fujimori, F., Takahashi, K., Uchida, C., Uchida, T., 1999. Mice lacking Pin1 develop normally, but are defective in entering cell cycle from G0 arrest. Biochem. Biophys. Res. Commun. 265, 658-663.

Gurova, K.V., Hill, J.E., Razorenova, O.V., Chumakov, P.M., Gudkov, A.V., 2004. p53 pathway in renal cell carcinoma is repressed by a dominant mechanism. Cancer Res. 64, 1951-1958.

Heimdal, K., Lothe, R.A., Lystad, S., Holm, R., Fosså, S.D., Børresen, A.L., 1993. No germline TP53 mutations detected in familial and bilateral testicular cancer. Genes Chromosom. Cancer 6, 92-97.
Liou, Y.C., Ryo, A., Huang, H.K., Lu, P.J., Bronson, R., Fujimori, F., Uchida, T., Hunter, T., Lu, K.P., 2002. Loss of Pin1 function in the mouse causes phenotypes resembling cyclin D1-null phenotypes. Proc. Natl. Acad. Sci. 99, 1335-1340.

Lu, K.P., Zhou, X.Z., 2007. The prolyl isomerase PIN1: a pivotal new twist in phosphorylation signalling and disease. Nat. Rev. Mol. Cell Biol. 8, 904-916.

Lu, P.J., Zhou, X.Z., Shen, M., Lu, K.P., 1999. Function of WW domains as phosphoserine-or phosphothreonine-binding modules. Science 283, 1325-1328.

Mantovani, F., Tocco, F., Girardini, J., Smith, P., Gasco, M., Lu, X., Crook, T., Del Sal, G., 2007. The prolyl isomerase Pin1 orchestrates $\mathrm{p} 53$ acetylation and dissociation from the apoptosis inhibitor iASPP. Nat. Struct. Mol. Biol. 14, 912-920.

Massagué, J., 2008. TGFbeta in cancer. Cell 134, 215-230.

Mazelin, L., Bernet, A., Bonod-Bidaud, C., Pays, L., Arnaud, S., Gespach, C., Bredesen, D.E., Scoazec, J.Y., Mehlen, P., 2004. Netrin-1 controls colorectal tumorigenesis by regulating apoptosis. Nature 431, 80-84.

O’Connor, P.M., Jackman, J., Bae, I., Myers, T.G., Fan, S., Mutoh, M., Scudiero, D.A., Monks, A., Sausville, E.A., Weinstein, J.N., 1997. Characterization of the p53 tumor suppressor pathway in cell lines of the National Cancer Institute anticancer drug screen and correlations with the growth-inhibitory potency of 123 anticancer agents. Cancer Res. 57, 4285-4300.

O'Hayer, K.M., Counter, C.M., 2006. A genetically defined normal human somatic cell system to study ras oncogenesis in vivo and in vitro. Methods Enzymol., 637-647. Elsevier.

Pang, R., Yuen, J., Yuen, M.F., Lai, C.L., Lee, T.K.W., Man, K., Poon, R.T.P., Fan, S.T., Wong, C.M., Ng, I.O.L., 2004. PIN1 overexpression and beta-catenin gene mutations are distinct oncogenic events in human hepatocellular carcinoma. Oncogene 23, 4182-4186.

Ryo, A., Nakamura, M., Wulf, G., Liou, Y.C., Lu, K.P., 2001. Pin1 regulates turnover and subcellular localization of -catenin by inhibiting its interaction with APC. Nat. Cell Biol. 3, 793-801.

Tuominen, V.J., Ruotoistenmäki, S., Viitanen, A., Jumppanen, M., Isola, J., 2010. ImmunoRatio: a publicly available web application for quantitative image analysis of estrogen receptor (ER), progesterone receptor (PR), and Ki-67. Breast Cancer Res. 12, R56.

Warburton, H.E., Brady, M., Vlatkovi, N., Linehan, W.M., Parsons, K., Boyd, M.T., 2005. p53 regulation and function in renal cell carcinoma. Cancer Res. 65, 6498-6503.

Winkler, K.E., Swenson, K.I., Kornbluth, S., Means, A.R., 2000. Requirement of the prolyl isomerase Pin1 for the replication checkpoint. Science 287, 1644-1647.

Wulf, G.M., Ryo, A., Wulf, G.G., Lee, S.W., Niu, T., Petkova, V., Lu, K.P., 2001. Pin1 is overexpressed in breast cancer and cooperates with Ras signaling in increasing the transcriptional activity of c-Jun towards cyclin D1. EMBO J. 20, 3459-3472.

Yaffe, M.B., Schutkowski, M., Shen, M., Zhou, X.Z., Stukenberg, P.T., Rahfeld, J.U., Xu, J., Kuang, J., Kirschner, M.W., Fischer, G., 1997. Sequence-specific and phosphorylation-dependent proline isomerization: a potential mitotic regulatory mechanism. Science 278, 1957-1960.

Yeh, E.S., Means, A.R., 2007. PIN1, the cell cycle and cancer. Nat. Rev. Cancer 7, 381-388.

Yeh, E., Cunningham, M., Arnold, H., Chasse, D., Monteith, T., Ivaldi, G., Hahn, W.C., Stukenberg, P.T., Shenolikar, S., Uchida, T., 2004. A signalling pathway controlling c-Myc degradation that impacts oncogenic transformation of human cells. Nat. Cell Biol. 6, 308-318.

Yeh, E.S., Lew, B.O., Means, A.R., 2006. The loss of PIN1 deregulates cyclin $\mathrm{E}$ and sensitizes mouse embryo fibroblasts to genomic instability. J. Biol. Chem. 281, 241-251.

Yi, P., Wu, R.C., Sandquist, J., Wong, J., Tsai, S.Y., Tsai, M.J., Means, A.R., O'Malley, B.W., 2005. Peptidyl-prolyl isomerase 1 (Pin1) serves as a coactivator of steroid receptor by regulating 
the activity of phosphorylated steroid receptor coactivator 3 (SRC-3/AIB1). Mol. Cell Biol. 25, 9687-9699.

You, H., Zheng, H., Murray, S.A., Yu, Q., Uchida, T., Fan, D., Xiao, Z.X.J., 2002. IGF 1 induces Pin1 expression in promoting cell cycle S phase entry. J. Cell Biochem. 84, 211-216.

Zacchi, P., Gostissa, M., Uchida, T., Salvagno, C., Avolio, F., Volinia, S., Ronai, Z., Blandino, G., Schneider, C., Del Sal, G.,
2002. The prolyl isomerase Pin1 reveals a mechanism to control p53 functions after genotoxic insults. Nature 419, 853-857.

Zheng, H., You, H., Zhou, X.Z., Murray, S.A., Uchida, T., Wulf, G., Gu, L., Tang, X., Lu, K.P., Xiao, Z.X.J., 2002. The prolyl isomerase Pin1 is a regulator of p53 in genotoxic response. Nature 419, 849-853. 